\title{
Constructivismo sistémico y diagnóstico organizacional
}

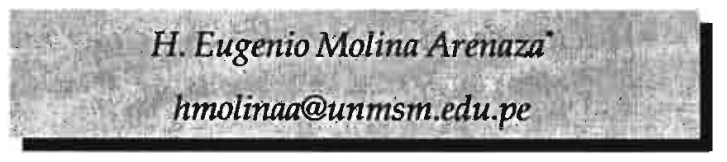

RESUMEN Los estudios realizados sobre el diagnóstico organizacional y cambio organizacional en las instituciones establecen que dicho diagnóstico está ligado al modelo del constructivismo sistémico. Del mismo modo el cambio paradigmático implicado por tal modelo en su desarrollo reciente conduce a cambios epistemológicos y los subsecuentes desafios para las metodologías de la investigación. Además, el enfoque sistémico aparece como una forma de dar respuesta a problemas derivados de la propia ciencia y que no pueden ser resueltos de la forma habitual que la ciencia tiene de enfrentar el conocimiento En estos apuntes de difusión se discuten algunos aspectos básicos relativos al diagnostico, junto a los cambios epistemológicos, y se ejemplifican algunos problemas de las técnicas de recolección - interpretación de la información en las ciencias sociales.

Palabras clave: Diagnóstico organizacional, cambio organizacional, enfoque sistémico.

\begin{abstract}
Studies undertaken over diagnostic and change organizational in the institutions establish that such diagnostic is linked to the model of the systemic constructivism. In the same way the paradigmatic change implied by such model in its recent development leads to epistemologies change and the subsequent challenger for the research methodologies. Moreover, the systemic approach appears as a form to give answer to problems derived from the own and that cannot be solved in habitual form that the science must to face the knowledge. In this notes of diffusions have been discussed some basic aspect relative to diagnostic together the epistemologists changes, and some problems of the techniques of recollection-interpretation of information in socials sciences are exemplified.
\end{abstract}

Keywords: Organizational diagnostic, change organizational, entropy - information.

\section{INTRODUCCIÓN: BASES DEL DIAGNÓSTICO}

La definición etimológica del término diagnóstico procede de dos palabras: día que significa a través y gnosis que significa conocer. Así, el diagnóstico es un instrumento metodológico que a partir de determinados métodos nos ayuda a reconocer 'e interpretar los problemas y dificultades más relevantes de un individuo, un grupo, un sector o un sistema.

El diagnóstico plantea el problema del conocimiento que involucra consideraciones epistemológicas básicas que son necesarias para realizar afirmaciones y recomendaciones sobre el funcionamiento y posibilidad de cambio en las organizaciones. En la medida que nuestros diagnósticos sean apropiados, nos llevarán a decisiones también apropiadas. El diagnóstico no sólo comprende el acopio de información, sino principalmente su análisis e interpretación cada vez que necesitemos tomar decisiones adecuadas acerca de situaciones o alternativas que cotidianamente enfrentamos. Hacemos un diagnóstico cuando pretendemos evaluar, anticipar o ponderar alternativas. Por ejemplo, cuando tratamos de:

\footnotetext{
* Economista. Profesor Auxiliar de la Facultad de Ciencias Administrativas de la UNMSM.
} 
- Evaluar diferentes líneas de acción (es el caso del empresario que pondera las posibles consecuencias en la diversificación de su producto).

- Coordinar los posibles resultados de nuestras acciones con otras personas o grupos (es el caso de los llamados acuerdos o alianzas organizacionales de toda índole).

- Identificar las causas de una determinada falla(es el caso del mecánico que trata de hallar las fallas de un motor).

- Anticipar las posibles consecuencias o reacciones motivadas por nuestras elecciones (es el caso de una familia, una empresa o un País que analiza la distribución de su ingreso o su presupuesto de inversión).

El diagnóstico tiene su origen en las ciencias médicas. Esta es la rama del conocimiento en donde el uso del diagnóstico se ha desarrollado con mayor profundidad analítica y diversidad técnica.

El diagnóstico de carácter social se ubica en otro nivel de conocimiento que con el apoyo de diversas técnicas en el acopio de información (cuantitativas cualitativas), obtiene resultados que son empleados para el diseño y ejecución de diferentes propuestas sociales.

En definitiva, el proceso de diagnóstico es iniciado por una consulta del interesado a un especialista, generando una situación social en que se desarrollan expectativas y se espera que el especialista sea capaz de interpretar la información dentro del marco de sus conocimientos y plantear soluciones al problema identificado. Las características de tal proceso son:

1. El diagnóstico es parte de una práctica profesional, donde un determinado especialista debe hacer uso de su conocimiento para interpretar las manifestaciones o síntomas del consultante interesado.

2. Se espera que el especialista sea capaz de relacionar la acumulación de sus conocimientos con la información que le entrega el cliente consultante, de tal manera que le permita considerar la información relevante y omitir la no relevante.

3. El diagnóstico es considerado parte de un proceso en el que se tomarán medidas a partir de esta primera fase diagnóstica.

4. Por consiguiente, el diagnóstico encuentra su sentido en su aplicabilidad práctica para solucionar uno o más problemas.
5. En general, el problema es experimentado por el paciente o el interesado que acude al especialista en busca de alguna solución.

6. El especialista, conociendo el funcionamiento del cuerpo humano, debe ser capaz de interpretar el malestar del paciente como indicadores, como guías.

7. En todo el proceso, el médico actúa como un "receptor activo", es decir, como un observador que activamente busca evidencias que le permitan encontrar las causa de los síntomas o mejorar su diagnóstico, pero que no influye en lo observado.

La mayor parte de las características mencionadas se pueden extender al diagnóstico organizacional. La diferencia consiste en que el consultante ya no es una persona, sino un sistema social (la organización).

Al final del proceso, el profesional podrá comunicarle a su consultante cuál es su problema, las causas de su problema e indicarle el tratamiento adecuado.

\section{EL SISTEMA ORGANIZACIONAL}

Las organizaciones constituyen sistemas sociales que se caracterizan porque:

a. Establecen condiciones que deben cumplir quienes deseen ingresar a ellas.

b. Ponen condiciones que deben ser satisfechas por todos los miembros, mientras permanezcan en ellas.

Las organizaciones constituyen una forma de construcción de sistema que surge a partir de la coordinación de dos contingencias. Contingente es algo que es como es, pero no tiene por qué serlo. Es decir, es algo no necesario, pero tampoco imposible. Por ejemplo, si en este momento usted esta leyendo este párrafo, es un hecho contingente, pues podría encontrarse haciendo algo diferente. Cada vez que se relacionan dos seres humanos se produce un acomodo entre dos contingencias. Los sistemas sociales requieren de esta doble contingencia para constituirse, dado que si uno u otro de los participantes decide optar por otra

alternativa distinta a la de estar allí, no podrá producirse el sistema social.

En las organizaciones, esta doble contingencia se traduce en:

L. La contingencia de los reglamentos y normas que la organización establece para regular los comportamientos de los miembros. 
II. La contingencia de los comportamientos de los miembros.

Tanto las reglas como los comportamientos pueden ser diferentes a lo que son, pero el hecho es que son como son. La organización que funciona en la práctica es la que resulta del acomodo entre reglas y comportamientos: ni las reglas se respetan como se hubiera deseado, ni los miembros se comportan conforme a lo previsto.

Aunque el estudio organizacional se inicia alrededor de 1911 y la conceptualización sistémica de las organizaciones sólo data de la década del sesenta, hoy prácticamente todos los enfoques de estudio del fenómeno organizacional reconocen una raíz en la teoría de sistemas. Además, hay que agregar que desde el comienzo mismo del Desarrollo Organizacional se utilizó un instrumental basado en la comprensión de las organizaciones como sistemas sociales y de sus fenómenos como procesos que ocurren en un contexto sistémico complejo.

El Desarrollo Organizacional ha mantenido una estrecha relación con la teoría de sistemas, de donde se desprende que ha ido experimentando cambios vinculados con la evolución de esta importante corriente teórica. Pero cabe indicar que como el Desarrollo Organizacional es una derivación práctica del marco conceptual teórico de esa teoría, sus modificaciones no han evolucionado al mismo ritmo que los cambios e innovaciones del modelo sistémico.

Algo parecido ocurre con la discusión epistemológica que, teniendo un lugar central en la construcción conceptual de la más moderna teoría de sistemas, sus influjos llegan sumamente atenuados a los oídos de los expertos y consultores de Desarrollo Organizacional.

\section{LA EPISTEMOLOGÍA}

La problemática epistemológica ha dejado de ser asunto exclusivo de los filósofos del conocimiento. Bateson (1976) demostró la importancia de la preocupación epistemológica en el quehacer cotidiano. En su conocida afirmación: "iYo soy la epistemología!" Bateson (además de remarcar la centralidad del observador en su configuración de lo conocido) destacó que la reflexión epistemológica resulta clave para comprender adecuadamente las relaciones que establecemos con otras personas y con el mundo. Por ejemplo; a partir del trabajo de Bateson se han desarrollado métodos y técnicas de terapia familiar sistémica que toman en consideración las relaciones intrafamiliares y las explicaciones que construyen los miembros del sistema familiar acerca de estas relaciones. Al respecto resulta interesante el modo en que estos dos niveles se entrecruzan, generando paradojas y contradicciones que conducen a situaciones de difícil salida, tales como las de doble vínculo estudiadas por Bateson, o las similares al caso del esquizofrénico que, según las observaciones de Watzlawick (1983), no quiere comunicar, pero está atrapado por la imposibilidad de no comunicar: incluso con su comportamiento retraído, prescindente y aislado comunica que no quiere comunicarse.

El planteamiento de Bateson (1976) se basa en el reconocimiento de la posición central del observador en la constitución de lo observado. En su conocido metálogo: "¿Por qué se desordenan las cosas?" demuestra en forma magistral que el orden está estrechamente relacionado con el observador de dicho orden: la pieza de una niña de cortos años, el ropero de un joven estudiante, el escritorio de un viejo usurero, pueden ser vistos como expresión perfecta y pura del caos y del reinado del azar y la equiprobabilidad para todos cuantos deseen entenderlos, con la excepción de sus propietarios, para quienes se trata de aglomerados perfectamente coherentes y ordenados.

La propuesta de Bateson quedó posibilitada por el trabajo de Wiener (1948), que vincula un problema de las ciencias naturales (la entropía) con uno de las ciencias humanas (la información). La entropía es el negativo de la información, de tal manera que a mayor información menor entropía. Esto quiere decir, según Wiener, que todos los casos de entropía están referidos al grado de conocimiento que se tenga acerca del sistema en que se da esta entropía.

Desde la moderna teoría de sistemas de Niklas Luhmann se puede relacionar el tema de las ciencias naturales (la complejidad) con el de las ciencias humanas (el sentido). Los sistemas sociales (y los de conciencia) reducen la complejidad mediante el sentido.

El modelo sistémico tiene pretensiones de universalidad lo que implica (entre otras cosas) que debe poder ser aplicado a sí mismo. En decir, el modelo sistémico ha experimentado un cambio epistemológico que va desde el perspectivismo a la autorreferencia. Se ha transformado en una epistemología que apunta a sí misma con el mismo instrumental que usa para tratar otros sistemas. Tal como lo indica SpencerBrawn (1979) se introduce o, reintroduce (re-entry) al sistema la distinción que permite diferenciar al sistema de su entorno. Las ciencias sociales puede distinguirse a sí mismas y constituirse como objeto a ser estudiado, así como también, hacer reflexiones acerca de la sociedad, desde dentro de la sociedad, vale 
decir, sin tener que postular una posición privilegia$\mathrm{da}$, externa, desde la cual sea posible describir los procesos sociales.

Con el diagnóstico organizacional, se trata de una evaluación del quehacer humano hecha por seres humanos. El problema de esta evaluación es el mismo que se presenta a todo intento científico en ciencias sociales: ¿Cómo evitar que interfieran los prejuicios y preferencias del observador con aquello que observa?

\section{EL RESPALDO DEL MÉTODO CIENTÍFICO}

Como se sabe, la respuesta no flota en el aire sino que se ancla firmemente en la tradición propia de las ciencias y de su quehacer. Esta respuesta sólida es el método científico.

El método tiene una importancia crucial en la ciencia. Gracias a él y a la rigurosidad de su aplicación, es que se hace posible diferenciar el conocimiento científico de otros modos de conocer.

El método que se ha definido como propio del pensamiento científico, tiene como fundamento la racionalidad analítica. El problema de la complejidad del mundo y de las limitaciones antropológicas para abarcarla, se resuelve por medio de una razón que es capaz de dividir y subdividir, para comprender y luego configurar lo así conocido.

La razón analítica obtiene resultados (en el pensamiento formal, matemático; en la política; en la economía; en la guerra; en la ciencia) y por esto pasa a transformarse la división en sinónimo de razón. Por ejemplo; la división del trabajo, puede ser llevada a extremos que no se producen espontáneamente, sino que son resultado de la aplicación a las tareas humanas de una "racionalidad científica", como lo intenta Taylor.

Además, el método debe permitir que el acto de conocer quede determinado por el objeto. Es decir, el conocer es un proceso interactivo entre un sujeto que conoce y un objeto que es conocido. En esta interacción es posible que el sujeto vea lo que quiere ver, o sea, que deje (consciente o inconscientemente) que sus preferencias y prejuicios alteren las características del objeto conocido. El método debe impedir esta interferencia de lo subjetivo en el conocimiento.

Resulta anecdótica la problemática así planteada donde la acción de conocer se define como una acción en la que el rol del objeto determina activamente y en la que al sujeto le quepa un rol pasivo. El conocer se transforma, así, en una suerte de padecer en que la acción del sujeto se limita a dejarse impresionar por el objeto.
Con el surgimiento del modelo sistémico, se produce un cambio en la aproximación al conocimiento $y$, por ende, en el método científico.

De hecho, el modelo sistémico tiene pretensiones de universalidad $y$, con ellas, se ve obligado a considerarse a sí mismo como parte de su objeto de estudio. Además, hay que tener en cuenta que la teoría de sistemas aparece como respuesta al descontento de algunos científicos con el método reduccionista que había imperado en las ciencias y que impedía captar lo propio de algunos ámbitos específicos de estudio, tales como los de la biología y los de las ciencias humanas, que surgía a partir de cierta complejidad irreductible, de un nivel dado de emergencia.

El modelo sistémico, como lo indica su nombre, tiene por objeto la comprensión de totalidades complejas, que deben ser entendidas en su globalidad y no de forma analítico - reduccionista.

El observador pasa a ocupar una posición central en este modelo. El sistema incluye al observador, de tal modo que el problema que se plantea es el de la autorreferencia. Toda observación que el observador haga es también parte del sistema e influye en él. El observador ya no puede pretender observar sin ser observado.

Bajo estas consideraciones, se hace necesario reconceptualizar el acto de conocer.

El sujeto es parte de su objeto y configura al objeto en el momento del conocimiento. Sujeto y objeto no pueden ser entendidos ya como entes separados, sino como integrados en el acto creador de conocer. Pero, ¿ cómo se entiende ahora la objetividad?

\section{LOS HECHOS, LA OBJETIVIDAD Y LOS MOLINOS DE VIENTO}

Se ha transformado en un lugar común el definir (y entender) la objetividad como adecuación entre una determinada aseveración y la realidad. Por ejemplo: se dice que una cierta explicación científica es objetiva, en la medida que se puede demostrar que en ella no ha tenido influencia el sujeto que conoce. Todo lo que ella afirma ha resultado, por consiguiente, de la observación fría de las características del objeto.

De esta forma, se llega a entender que en la ciencia se explica la realidad y que esta explicación resulta mejor que otras como, por ejemplo, las del conocimiento vulgar, porque el método científico permite asegurar la objetividad, entendida como reflejo fiel de la realidad. 
Según Goode y Hatt (1952), lo que la ciencia estudia son hechos y éstos son observaciones empíricamente verificables. Ellos, remarcan que la ciencia es un método de aproximación al mundo empírico, es decir, al mundo susceptible de ser captado por el hombre a través de su experiencia.

Hablamos que los hechos son porfiados, que se resisten a la manipulación y a los deseos de quienes los observan y en esta característica de los hechos, se fundamenta la exigencia para la ciencia de tener un carácter empírico, vale decir, que sus afirmaciones sean comprobables a través de la experiencia.

Desde el origen mismo de las ciencias sociales encontramos que sus fundadores (Durkheim Comte) consideraban necesario que para que éstas adquieran el nivel científico, se debe adoptar como propio el método científico, que en esa época era el positivista. El positivismo sostiene que es imposible tener acceso al mundo real. En consecuencia, sólo es posible basar el conocimiento en lo perceptible, en el mundo sensible, aquel que podemos captar a través de nuestros sentidos. Por consiguiente, un hecho "positivo" surge en la observación y es obtenido mediante los sentidos.

Dentro de esta perspectiva, el problema de Durkheim consiste en establecer una ciencia de lo social con basamento empírico, es decir, cuyas afirmaciones se basen en datos inmediatos, empíricamente comprobables.

La objetividad debería entenderse, entonces y ahora, como adecuación entre explicación científica y hecho; como verificabilidad empírica de las observaciones y aseveraciones hechas en el marco de la ciencia. Asimismo, el método debería permitir asegurar la correspondencia entre lo afirmado y la experiencia. Pero, este no es el caso. La epistemología subyacente a la postura positivista y a la posición que actualmente prevalece entre los científicos, supone la existencia de una realidad a la que sólo podemos tener acceso mediante nuestros sentidos. Esta postura epistemológica es válida, pero de ella se desprende (innecesariamente) la afirmación de que la comprobación de la objetividad de un aserto científico se logra mediante su mejor aproximación a la realidad.

La comprobación de la objetividad que se logra mediante un plus en la aproximación a la realidad es innecesario, pero se ha transformado en el símbolo por el que quiebran lanzas los caballeros de laciencia; defendiendo o atacando los molinos de viento de una realidad, de la cual tenemos una experiencia que es innegable, pero para la ciencia está vedado cualquier otro camino, cualquier atajo directo que nos conduzca a la realidad.

\section{SER OBJETIVOS: HABLANDO DE LA "OBJETI- VIDAD"}

A partir del modelo sistémico y, particularmente desde la biología, se ofrece una redefinición del método científico que (aunque permite dejar en claro la centralidad de la experiencia) ha provocado muchas polémicas.

El destacado biólogo Humberto Maturana (1990) es quien plantea esta redefinición remarcando que en la ciencia no se explica la realidad, sino la experiencia. El conocimiento ya no puede ser validado por nada externo al observador. Luego no se puede recurrir a la realidad externa como criterio de validación de las explicaciones científicas. Según Maturana, hay dos formas de validar una explicación científica cualquiera:

1. Recurriendo a la realidad externa, independiente del observador, de tal modo que será válida una afirmación que logre demostrar que es un reflejo fiel de esta realidad. Este es el camino de la objetividad.

2. Aceptando la centralidad del observador en la construcción de lo observado. Esto implica "poner la objetividad entre comillas", que no es lo mismo que subjetividad, sino simplemente desechar el argumento de la realidad externa, independiente del observador, como criterio de validación. La validación se hace entonces haciendo referencia a la experiencia. Este es el camino de la "objetividad". Sin embargo, esto nos lleva a un problema adicional, que es el punto de partida de la reflexión del propio profesor Maturana. Sus trabajos en biología experimental lo llevaron a constatar que es imposible (en el plano de la experiencia) distinguir entre ilusión y percepción.

Esta constatación conduce al mismo problema al que hemos estado aludiendo: la imposibilidad de recurrir a una adecuación con la realidad externa como forma de validación de las afirmaciones científicas.

Por consiguiente, es necesario redefinir los criterios de validación de las afirmaciones científicas. Estos criterios deben asumir que lo que se explica es la experiencia y, además, deben permitir la acumulación científica por medio de la comunicabilidad del conocimiento y su réplica por otros observadores en el ámbito de la ciencia.

El criterio propuesto por Maturana (1989) consiste en cuatro operaciones que, si son satisfechas cabalmente, permitirán que una explicación pueda ser aceptada como explicación válida en el dominio de las explicaciones científicas. 
1. Descripción del fenómeno a explicar en términos de lo que el observador debe realizar para tener la experiencia del fenómeno que se quiere explicar.

2. Hipótesis explicativa, que consiste en la proposición de un mecanismo que si el observador lo hace operar, entonces le permitirá experimentar(en su dominio de experiencias) el fenómeno que se quiere explicar.

3. Deducción (a partir de la operación del mecanismo generativo o hipótesis explicativa) de otras experiencias que deberían surgir en el dominio de experiencias del observador y de las operaciones que el observador debería realizar para experimentarlas: "si ocurre B, debería ocurrir $X$ en tales y cuales condiciones".

4. La realización de las experiencias deducidas por el observador, mediante la satisfacción de las operaciones indicadas en la operación 3.

El criterio de validación así expuesto, no recurre a la realidad objetiva, sino simplemente a la experiencia, al fenómeno, y no a la cosa en sí. Por lo tanto, el método científico es reformulado. La "objetividad" deja de ser entendida como adecuación al objeto, para pasar a ser entendida como adecuación a un conjunto de criterios de validación aprobados por la comunidad de observadores.

La propuesta de Maturana constituye una redefinición de gran importancia para el quehacer científico. En efecto, la rigurosidad propia de la investigación en la ciencia adquiere mayor relevancia por el carácter fenomenológico de la explicación científica. La responsabilidad del investigador se hace inevitable dado que ya no puede responsabilizar de sus errores a un objeto externo, sino a su propia experiencia o a sus explicaciones de ésta. Asimismo, las explicaciones no reemplazan las experiencias que explican. Unas y otras tienen lugar en dominios fenoménicos distintos dado que una explicación es una reformulación (en el dominio explicativo) de la experiencia.

De otro lado, en el caso de las ciencias sociales, el problema es conocido y además presenta dos aristas difíciles de ser abordadas.

a. Se trata de un problema conocido porque desde su misma incorporación al mundo científico las ciencias sociales han debido enfrentarse al problema de la participación del observador en la configuración de lo observado por él.

Tanto el esfuerzo de Weber como el de Durkheim tienen su raíz en la búsqueda de una solución a este problema de construir las bases para una ciencia "objetiva", con un observador conocedor de la alta probabilidad de que sus propios valores y perspectivas influyan en lo observado.

b. Es además, un problema difícil, por cuanto el objeto de estudio da (también) una explicación acerca de su experiencia y que las explicaciones (y las explicaciones de las explicaciones) constituyen también parte del objeto de estudio de las ciencias sociales. Este es el problema de la "doble hermenéutica" inherente al estudio de lo social, del cual habla Giddens (1982).

El objeto de estudio es un observador, que observa su mundo, que observa a otros observadores $y$ que se da explicaciones acerca de su estar en este mundo con otros observadores.

No obstante, si se comprende la observación como una operación en que se aplican esquemas de distinción que permiten hacer surgir lo observado sobre el trasfondo de su entorno y se entiende que el observador puede ver con sus esquemas de distinción, se tendrá una nueva perspectiva para aproximarse al fenómeno.

De hecho, "la observación de la observación" permitirá ver lo que los "observadores observados" no pueden ver: sus esquemas de distinción. Pero siempre quedará algo sin ser visto por el observador de los sistemas observados: sus propios esquemas de distinción.

\section{EL DIAGNÓSTICO ORGANIZACIONAL}

El diagnóstico de las organizaciones es un proceso en que un determinado observador explicará las experiencias que tiene de una organización y de su operar. Para ello utiliza sus esquemas de distinción, que le permiten destacar algo con respecto a un trasfondo. Por ejemplo, según cuál sea nuestro esquema de distinción, podemos distinguir, una persona, un grupo, o una colectividad. Asimismo, de acuerdo a los esquemas de distinción que usemos, en una misma persona podemos distinguir un hombre, un médico, un padre, un hijo, un esposo, un consumidor, etc.

Empero, en el diagnóstico organizacional no sólo es posible distinguir diferentes niveles, como destacar sus miembros, o los subgrupos internos, o los subsistemas y departamentos, o ver a la organización diferenciándose en relación con su entorno, sino que el observador al observar los diferentes procesos que tienen lugar en la organización puede distinguir 
el poder, las comunicaciones, la toma de decisiones, etc. Además, puede orientar la indagación diferenciando estructuras de proceso o factores ligados al diseño y los relacionados a las actitudes de los participantes en la organización. En general, el diagnóstico organizacional supone la aplicación de criterios o esquemas de distinción en una observación configuradora de lo observado para trazar los límites de la organización (o subsistema dentro de ésta).

El diagnóstico organizacional constituye una descripción, una explicación realizada por el observador para dar adecuadamente cuenta del operar de una organización determinada y al mimo tiempo permitir que otro observador pueda ser testigo (en su ámbito de experiencia) del funcionamiento organizacional descrito. Es decir, los procesos organizacionales deben ser generados a partir de la explicación. Y, desde ella deberán deducirse otros fenómenos observables en el ámbito de experiencia del observador. De este modo, el diagnóstico puede servir como instrumento válido en la comunicación científica y para que a partir de él se pueda implantar un proceso de cambio social. Por lo tanto, el diagnóstico de una organización debe cumplir con los criterios de validación de las explicaciones científicas.

Es importante tener en cuenta que una organización es un sistema social y, como tal, también tiene un autodiagnósico de su situación. En ese sentido el diagnóstico organizacional constituye un caso de investigación en que se produce la doble hermenéutica planteada por Giddens. Esto significa, que las explicaciones que los propios involucrados dan acerca de la organización y de su estar y actuar en ella, forman parte importante del objeto de estudio.

Un punto central a considerar es que el diagnóstico organizacional deberá siempre ser un codiagnóstico, en que el consultor externo colabore con consultor interno a la organización en la determinación conjunta de los problemas y las alternativas de solución de la organización. Este punto se justifica debido a que en el diagnóstico (como en toda observación) el observador al hacer uso de esquemas de distinción, puede configurar el objeto observado (algún proceso, subsistema o fenómeno organizacional), pero en donde ninguna observación agota todas las posibilidades de observación. En todo caso se trata de una perspectiva, y por lo tanto, existen aspectos que permanecen invisibles para el observador. En toda observación existe un punto ciego que el propio observador no puede ver, pero que puede ser visto por un observador de la observación el que, al mismo tiempo, no será capaz de ver su propio punto ciego.

\section{CONCLUSIONES}

El constructivismo sistémico incorpora la cibernética de segundo orden a partir de la cual es posible entender la diferencia sujeto - objeto ya no en términos ontológicos, sino en términos de que las operaciones de los "sujetos" quedan mejor comprendidas si se las entiende como surgidas en las observaciones, es decir, si entendemos al sujeto como "observador".

El problema de la observación de la observación es, en consecuencia, el de ver lo que el sistema observador observado ve $y$, además, lo que él no ve: sus esquemas de distinción.

El problema que debemos enfrentar es el de las técnicas de recolección de la información que permitirán este método. Sin pretender agotar el tema, sino sólo dejarlo planteado para su discusión se ha tomado el caso del diagnóstico en los sistemas organizacionales.

En el estudio de las organizaciones se hace uso de una metodología escalonada que va de lo más general y espontáneo a lo más particular y dirigido. Se genera, por lo tanto, una especificación creciente de temas y contenidos, a partir de los resultados de los instrumentos y procedimientos que se han delineado.

En una primera instancia, se realizan entrevistas (individuales y grupales) semiestructuradas, que pretenden abrir la discusión sobre la organización y donde van surgiendo paulatinamente las explicaciones en el lenguaje que los miembros de la organización dan de su estar en ella.

El asombro experimentado por el observador muestra (como lo indica Schein) los esquemas de distinción utilizados por los observadores observados. Se puede ver la contingencia de estos esquemas de distinción precisamente en el hecho de que (a pesar de resultar sorprendentes) han funcionado para los observadores observados y siguen siendo válidos para ellos.

Una vez identificados los esquemas de distinción utilizados por el colectivo, se hace necesario comprobar si éstos corresponden efectivamente a esquemas difundidos en el colectivo (y no a simples subgrupos o a atribuciones hechas por el observador). Para esto, se efectúan cuestionarios en que se pone a prueba la difusión de los conceptos. Estos cuestionarios son cerrados y sus ítems surgen de las entrevistas grupales e individuales.

Para comprobar si la interpretación que el observador ha hecho de la interpretación de los 
observadores observados es adecuada, se construyen además cuestionarios con historias laborales y con valores descriptivos. En ellos se trata de poner a prueba las interpretaciones, pero con un campo delimitado de alternativas.

Esta metodología ha resultado de gran utilidad para el estudio de culturas organizacionales, que (como toda cultura) tienen la característica de no resultar fácilmente accesibles a la observación de los observadores inmersos en ellas.

Una cultura constituye las gafas a través de las cuales configuramos el mundo, pero a las cuales no vemos. Un observador externo, en cambio, podrá ver las gafas que ocupamos y (también) podrá entender porque vemos el mundo del color que lo vemos. Empero, él no podrá ver sus propios anteojos.

Con esta metodología se busca permitir que los miembros de la organización describan su mundo (como ellos la ven), y al mismo tiempo, muestren sus esquemas de distinción.

Con el objeto de evitar una interpretación de la interpretación que pretenda erigirse como válida y superior, es necesario que el equipo consultor se haga asesorar por un consultor interno a la organización, que podrá ayudar a la interpretación.

Con esto no se conseguirá otra cosa que el intercambio de perspectivas, que permitirá poner en común un nuevo nivel de observación. Empero, también esta observación encuentra sus propios límites en sus invisibles esquemas de distinción.

En los últimos años se ha evidenciado una creciente preocupación por el estudio de los sistemas sociales entendidos como redes de conversaciones. Entre otros científicos sociales cabe destacar el estudio de Maturana y su noción de conversación como un entrelazamiento del lenguajear y emocionar. Según él, todo quehacer humano se da como un conversar en el que se coordinan haceres y emociones. Este interés encuentra su origen en la cibernética, la teoría de sistemas, la teoría del lenguaje y la biología del conocimiento.

Empero, en estas metodologías está siempre presente una disputa que (a pesar de su antigüedad) no se resigna a abandonar la escena. Esta es la disputa entre el subjetivismo y el objetivismo. En verdad, aunque en términos teóricos es absolutamente claro que la propuesta del modelo sistémico, en la versión de Maturana, o en la Luhmann, no lleva al subjetivismo (la "objetividad") no es un tipo de subjetivismo, sino que implica tomar el rol constitutivo que tiene la observación y el lenguaje, en algunas de las metodologías propuestas la opción parece ser claramente hacia un retorno al sujeto. Sin embargo, Maturana dice explícitamente que el sujeto - observador surge en las distinciones del observador y no es, de ninguna manera, una entidad trascendente.

Una metodología que reconozca que en la comunicación se requiere de Alter y Ego, y que por consiguiente la mínima unidad comunicacional es una síntesis de tres elecciones (un contenido, una expresión y una comprensión) podrá, llevar a evitar que de la objetividad se salte (junto con el péndulo) a la subjetividad.

Esta metodología debería permitir que se cumplieran las cuatro características del método de Maturana y, en definitiva, debería conducir a la generación de observaciones que pudieran ser aceptadas como válidas por la comunidad de científicos.

\section{BIBLIOGRAFÍA}

\section{BATESON (1976). En Revista Cinta de Moebio.}

BENNIS WARREN G. (1973). Desarrollo Organizacional: Su Naturaleza, sus Orígenes y Perspectivas. Fondo Educativo Interamericano, S.A., Bogota.

COLLINS, David (1996). "New Paradigms for Change?: Theories of Organization and the Organization of Theories". Journal of Organizational Change Management, Vol. 9, N. ${ }^{\circ} 4$, pp. 9-23.

CORSI, Giancarlo; ESPOSITO, Elena; BARALDI, Claudio (1996). "Glosario sobre la teoría social de Niklas Luhmann". Anthropos - Universidad Iberoamericana - Iteso, México.

GIDDENS (1982). En Revista Cinta de Moebio.

GOODE y HATT (1952). En Revista Cinta de Moebio.

MARCELO, Arnold (1998). "Recursos para la Investigación Sistémico Constructivista". En Revista Cinta de Moebio, $\mathrm{N}^{\circ} 3$.

MATURANA (1989-1990). En Revista Cinta de Moebio.

NIKLAS, Luhmann (1996). Sistemas Sociales. Anthropos - Universidad Iberoamericana - Iteso, México.

RODRÍGUEZ, Darío (1997). Diagnóstico Organizacional. Pontificia Universidad Católica de chile, Santiago.

SCHEIN, Edgar (1988). La Cultura Organizacional y el Liderazgo, Plaza Janés.

SPENCER-BRAWN (1979). En Revista Cinta de Moebio.

WATZLAWICK, Paul (1981). ¿Es Real la Realidad? Herder, Barcelona. 